\title{
The Gattini South Pole UV experiment
}

Anna M. Moore

Sara Ahmed

Michael C. B. Ashley

Ernest Croner

Alex Delacroix

Yusuke Ebihara

Jason Fucik

D. Christopher Martin

Viswa Velur

Allan Weatherwax 


\title{
The Gattini South Pole UV Experiment
}

\author{
Anna M. Moore*a, Sara Ahmed ${ }^{\mathrm{b}}$, Michael C. B. Ashley ${ }^{\mathrm{c}}$, Ernest Croner ${ }^{\mathrm{a}}$, Alex Delacroix ${ }^{\mathrm{a}}$, Yusuke \\ Ebihara $^{\mathrm{d}}$, Jason Fucik ${ }^{\mathrm{a}}$, D. Christopher Martin ${ }^{\mathrm{b}}$, Viswa Velur ${ }^{\mathrm{a}}$, Allan Weatherwax ${ }^{\mathrm{e}}$ \\ ${ }^{a}$ Caltech Optical Observatories, 1200 E California Blvd, Pasadena, CA, USA 91125; \\ ${ }^{\mathrm{b}}$ California Institute of Technology, 1200E California Blvd, Pasadena, CA, USA 91125; \\ ${ }^{\mathrm{c}}$ School of Physics, University of New South Wales, Sydney NSW 2052, Australia; \\ ${ }^{\mathrm{d}}$ Research Institute for Sustainable Humanosphere, Kyoto University, Kyoto, Japan; \\ ${ }^{\mathrm{e}}$ Department of Physics and Astronomy, Siena College, Loudonville, New York, USA.
}

\begin{abstract}
The Gattini South Pole UV experiment (Gattini SPUV) was deployed to the South Pole dark sector in February 2010 and has recently completed a highly successful first season of winter time observations. The experiment has, for the first time ever, measured and categorized the optical night sky brightness at the very blue wavelengths. The experiment consists of a remotely operated 6" aperture custom designed telescope. The telescope feeds a blue sensitive imager with 4 degree field of view that contains a bank of 3 filters: SDSS g', Bessel U and a custom "super U" filter specifically designed to probe the sky emission at wavelengths approaching the atmospheric cut-off. The filters are continually cycled with exposure times ranging from 30 to 300 seconds throughout the winter period. The telescope, in addition, feeds a 2 degree long slit VPH grating spectrograph with $\mathrm{R} \sim 1000$. The bandwidth is $350-450 \mathrm{~nm}$. The spectra are recorded simultaneously with the imager exposures. The experiment is designed for low temperature Antarctic operation and resides on the roof of the MAPO building in the South Pole Antarctic sector. The primary science goals are to categorize the Antarctic winter-time sky background at the very bluest of wavelengths as a pathfinder for the Antarctic Cosmic Web Imager. We present a technical overview of the experiment and results from the first winter season.
\end{abstract}

Keywords: Gattini, South Pole, Antarctic wide field surveys, cosmic web, site testing, cloud cover, aurora, night sky brightness, UV astronomy

\section{INTRODUCTION}

\subsection{Science Rationale}

The Gattini SPUV experiment will characterize the South Pole winter sky from 2011 onwards in the Astronomical U and SDSS g' bands to provide a foundation for future larger-scale experiments such as the Antarctic Cosmic Web Imager (direct detection of Lya emission of the Intergalactic Medium (IGM)) [1]. Such experiments require exquisite sky subtraction in the optical passbands to reach the estimated surface brightness magnitudes of the extended emission regions. A South Pole observing location provides a possible three-fold advantage for this science case over a temperate site: (i) a constant target elevation hence constant air mass, (ii) zero instrument flexure with a fixed pupil during an observation and (iii) a relatively continuous observing period lasting several months. The existing large scale infrastructure offered by the US Amundsen Scott station provides a logistically favorable target site for this collaboration on the high Antarctic plateau. The South Pole, however, is particularly prone to aurora that can be extremely bright in the optical passbands. The Gattini SPUV was designed to quantify the impact of this and other sky emission on the ability of a $2 \mathrm{~m}$ class telescope such as ACWI to directly detect the cosmic web signal.

*amoore@astro.caltech.edu

Ground-based and Airborne Telescopes IV, edited by Larry M. Stepp, Roberto Gilmozzi, Helen J. Hall, Proc. of SPIE Vol. 8444, 84441Q · @ 2012 SPIE · CCC code: 0277-786/12/\$18 - doi: 10.1117/12.927313 


\subsection{Objectives}

The primary objectives of the experiment are to:

1. Characterize the South Pole winter sky in the Astronomical U and SDSS g' bands for the first time to provide a foundation for future larger-scale experiments such as the Antarctic Cosmic Web Imager (direct detection of Lya emission of the Intergalactic Medium (IGM));

2. Produce a light curve dataset in the U and SDSS g' passbands to compliment the 6" CSTAR experiment at Dome A [3];

3. Quantify the effect of at least two of the brightest airglow lines in the U and SDSS g' bands;

4. Quantify the effect of at least two of the brightest auroral lines in the U and SDSS g' bands.

\subsection{Instrument Flowdown}

The technical specifications that meet the primary objectives are as follows

1. Gattini SPUV contains a 6" aperture telescope feeding a 4 degree field of view imager channel;

2. The telescope feeds a 2 degree long-slit spectrograph channel to permit simultaneous imaging and spectroscopy;

3. The imager channel contains a filter wheel with Bessel U, SDSS g' modified to exclude the 557.1nm OI emission line and a custom non-Astronomical "Super U" filter to probe the sky brightness at the very bluest of wavelengths the transmission of which is limited by atmospheric absorption and;

4. The spectrograph channel has a resolution of $\mathrm{R} \sim 400$ (capable of up to $\mathrm{R} \sim 2000$ with narrower slit) with a fixed passband of $350 \mathrm{~nm}-450 \mathrm{~nm}$.

In addition:

5. To reduce costs the telescope is stationary with no field de-rotation implemented;

6. Observations must be continuous with little or no overhead associated with downtime;

7. A minimum of two austral winter seasons of observation and;

8. The system is designed to permit operation at redder wavelengths with easy modification such as a manual grating or filter change during the austral summer.

\section{EXPERIMENT DESIGN}

The goal opto-mechanical design is discussed in detail in an existing article [2]. An update is presented here with additional discussion on the assembly, installation and performance.

\subsection{Optical layout}

The optical layout of the Gattini-UV design is shown in Figure 1. The system aperture is 6in diameter and the assembly fits inside a volume of $\sim 35 \times 35 \times 24 \mathrm{in}^{3}\left(0.9 \times 0.9 \times 0.6 \mathrm{~m}^{3}\right)$. The optical design consists of: (1) a 6 in aperture Telescope based on a Schmidt design with aspheric corrector plate that images $4^{\circ} \times 4^{\circ}$ field at Cassegrain focus; (2) the focus contains 2 fold mirrors - one to divert light to the imager and one to send a slit image to the spectrograph as shown in Figure 2; (3) for the imager channel a fast f/1.53 Schmidt camera images the $4^{\circ} \times 4^{\circ}$ image to the Andor CCD; (4) the imager channel contains a simple spherical collimator for imaging of the pupil at the 4-position filter wheel; (5) the spectrograph channel incorporates a slightly slower Schmidt camera for reimaging a $2^{\circ}$ long slit to the Andor CCD that is spectrally dispersed at a resolution of up to $\mathrm{R} \sim 2000$ depending on the slit width; (6) a static Volume Phase Holographic grating replaces the filter wheel in the spectrograph channel.

The secondary obscuration is $\sim 25 \%$. There are 15 optical elements of which 3 are aspheric, 3 are conic and the remaining items are either flat or spherical. A tolerance analysis was performed and incorporated into the mechanical design of the experiment. The theoretical imaging performance is excellent over the field with 2-pixel sampling of the PSF across the $4^{\circ}$ field of view. Distortion is low at $0.06 \%$. 


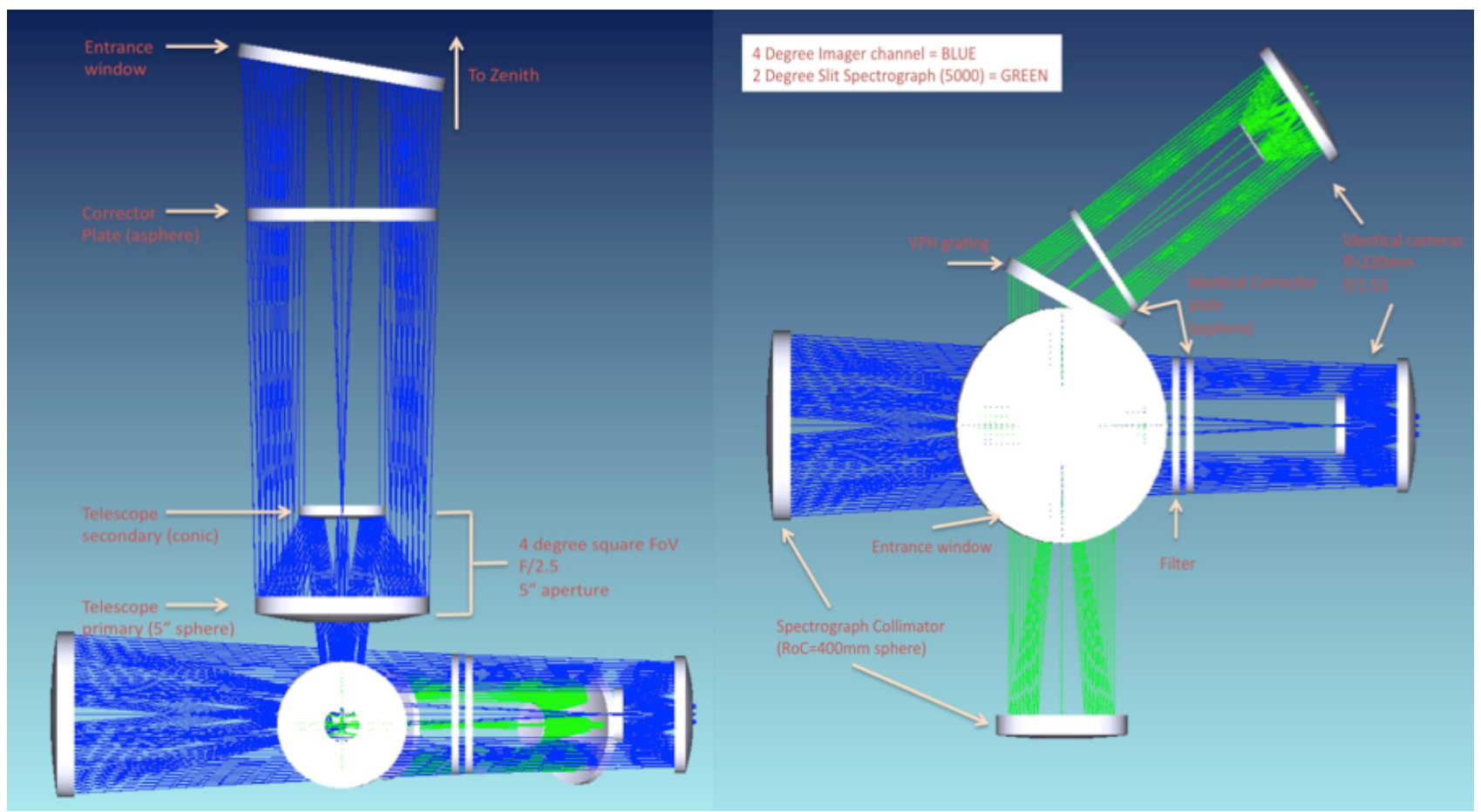

Figure 1: Optical layout of the double channel Gattini-SPUV experiment is shown in side view (left) and plan view (right).

All optics, refractive or otherwise, are made of UV grade fused silica. Fused silica has excellent transmission at UV wavelengths and, in addition, has a low thermal coefficient of expansion.

\section{Filter set}

The filter set contains two Astronomical filters (Bessel U and SDSS g') and one custom filter. The latter filter is coined "Super U" with a peak transmission at 320nm. The trabsmission curves of all three filters are shown in Figure 3.

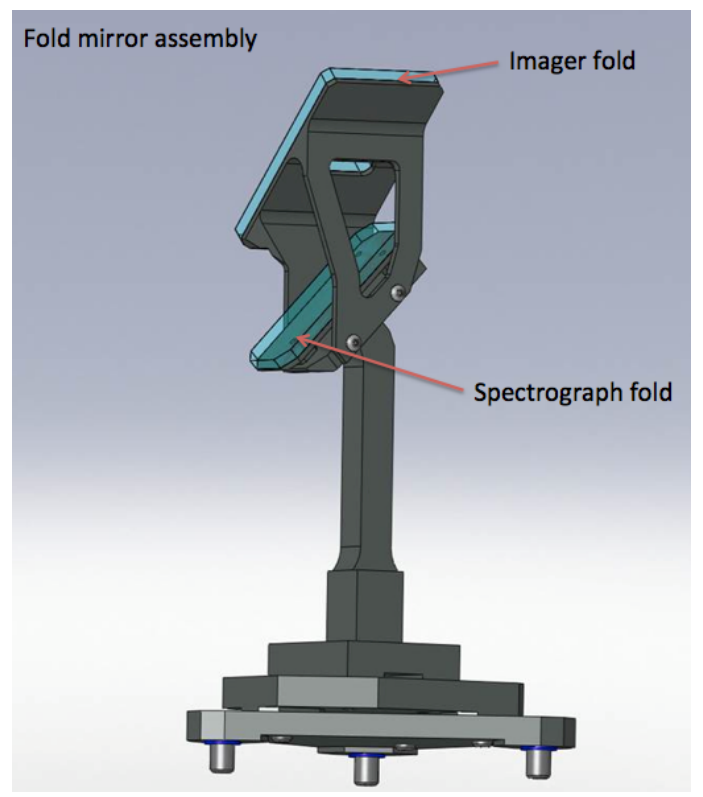

Figure 2: The fold mirror assembly supports the imager and spectrograph fold mirrors and provides the method for field separation between the two channels. 

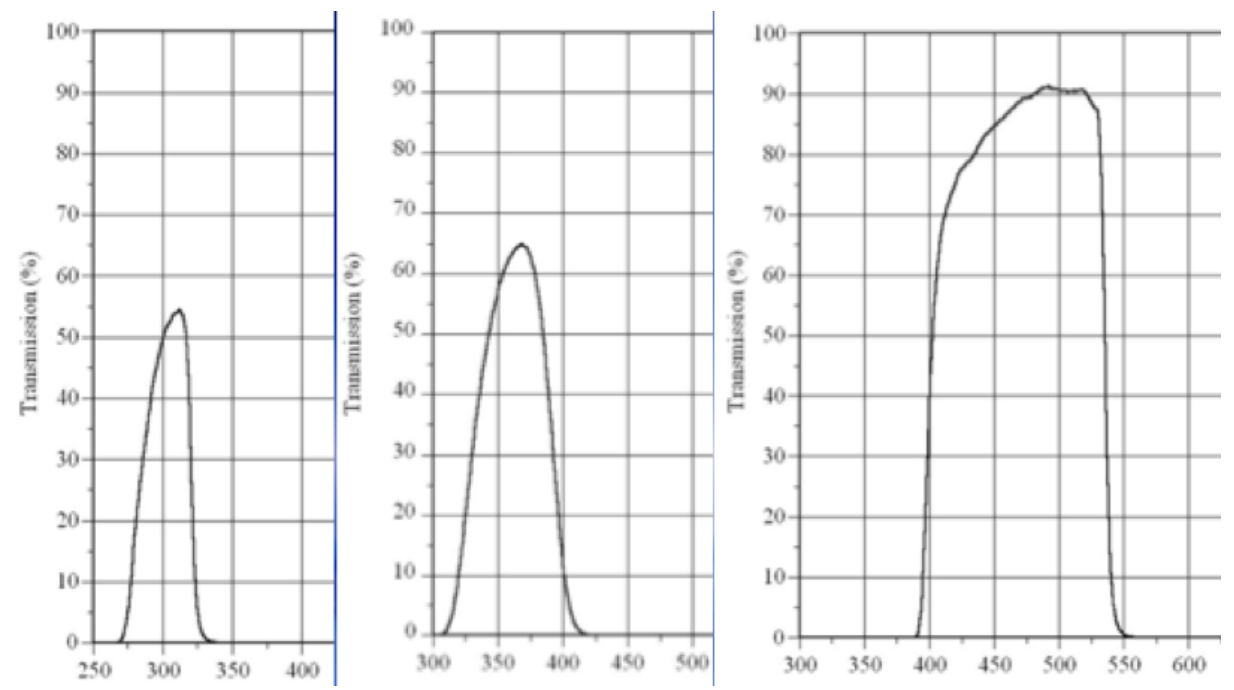

Figure 3: The transmission curves of the Gattini SPUV filter set, (left) the custom "Super U” filter, (center) Bessel U and (right) SDSS g' modified slightly to completely reject the $557 \mathrm{~nm}$ OI line.

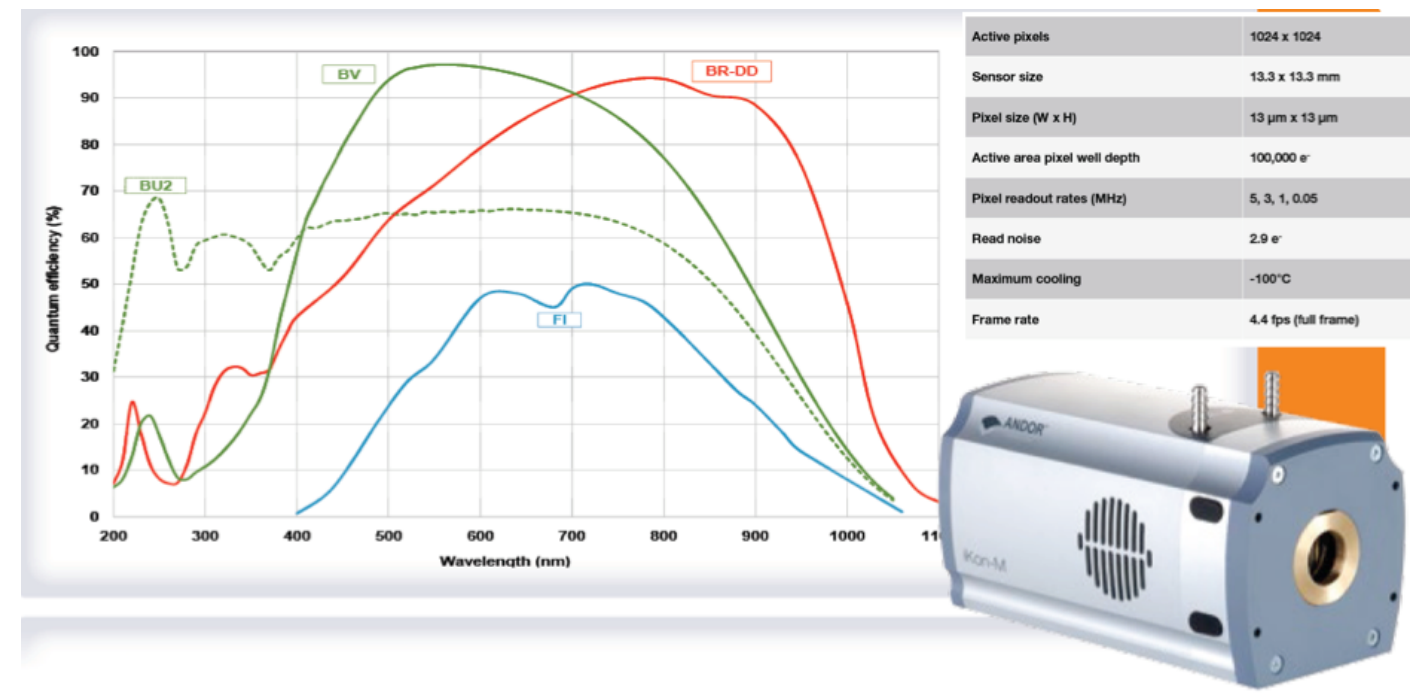

Figure 4: The "BU2" Andor Ikon-M model (lower right) was selected for both the imager and spectrograph channels. The associated specifications are shown (upper right) and quantum efficiency (left) were taken from the vendor website.

\subsection{CCD camera}

The imager and spectrograph channels adopt the same CCD camera model, the iKon-M 934 with "BU2" coating from Andor Technology shown in Figure 4. The CCD is a $1 \mathrm{k} \mathrm{x} 1 \mathrm{k}$ back-illuminated device with 13 um pixels. The 2-stage peltier cooler obtains a minimum detector temperature of $-80^{\circ} \mathrm{C}$ without water-cooling. Both cameras were performance tested prior to deployment at Caltech at a range of ambient temperatures down to a minimum of $-80^{\circ} \mathrm{C}$. The solenoidactivated shutter was extensively cold temperature tested during the verification phase at Caltech prior to deployment.

The cameras exhibited excellent performance with stable detector temperature, linear response over a large range of illumination, low read noise and, with regards to the shutters, perfect reliability. 


\subsection{Mechanical}

\section{Working temperature}

The operating temperature range for the Gattini-UV enclosure is $+25^{\circ} \mathrm{C}$ (lab) to $-75^{\circ} \mathrm{C}$ (minimum at South Pole). The control electronics are housed in a heated laboratory while operating at the South Pole so does not need to sustain the above temperature minimum.

\section{Opto-mechanical assembly}

A mechanical rendering of the opto-mechanical assembly is shown in Figure 5. The large baseplate and several of the critical mounts are machined from invar material to satisfy the tight optical tolerance requirements. The filter wheel mechanism incorporates a cryogenic rated stepper motor and limit switches. The filter mechanism was temperature tested successfully at Caltech prior to deployment.

\section{Enclosure}

The enclosure, shown in Figure 6, is made from sheet metal and is not completely hermetic. Care was taken to seal the enclosure as much as possible and over pressurize with dry Nitrogen prior to installation on the building roof at the South Pole in an attempt to minimize the amount of water forming on the optics as the internal air cooled. No evidence of ice formation on the optics has been seen in almost two years of operation so far. The entrance window is heated to prevent ice formation and has worked very well.

\subsection{Electrical}

The Gattini-UV enclosure, located at external ambient temperature, is linked to a rack-mounted control computer by necessary low temperature rated cables. This is the design layout adopted in all previous Gattini camera systems. MILSPEC connectors are used throughout the design, and rated cabling (PTFE) is used throughout the design. The University of New South Wales provided the control system in late November 2010.

\subsection{Assembly and testing prior to deployment}

The instrument was assembled under clean room conditions at Caltech in November 2010, as shown in Figure 7. The filter wheel and cameras were placed in a low temperature test chamber for several weeks prior to assembly onto the main optical bench. Each optical assembly was pre-tested with a collimated beam with optics adjusted to optimize the image quality.

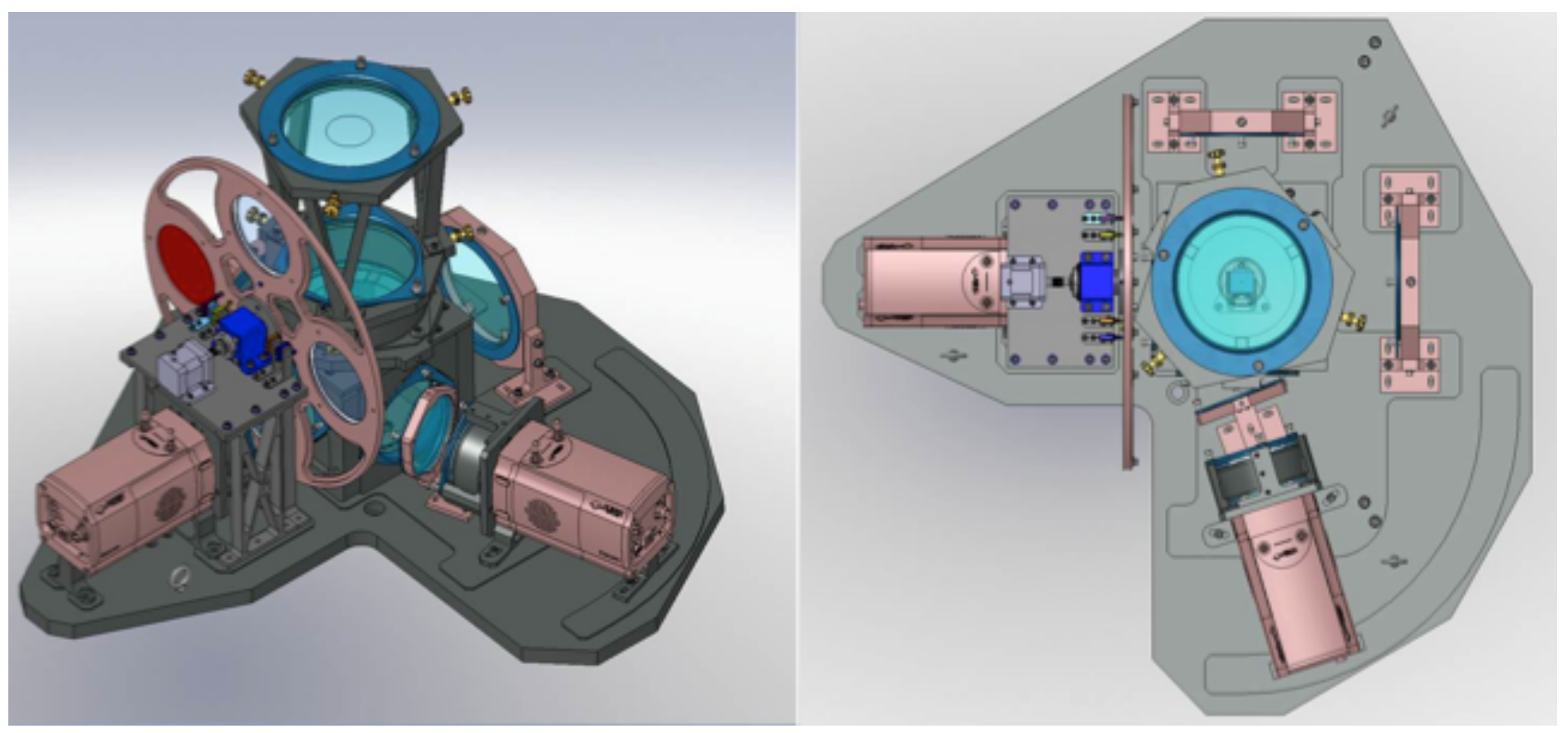

Figure 5: A mechanical rendering of the optical bench is shown in isometric view (left) and in plan view (right). The bench and several of the critical mounts are made of invar for increased thermal stability. 


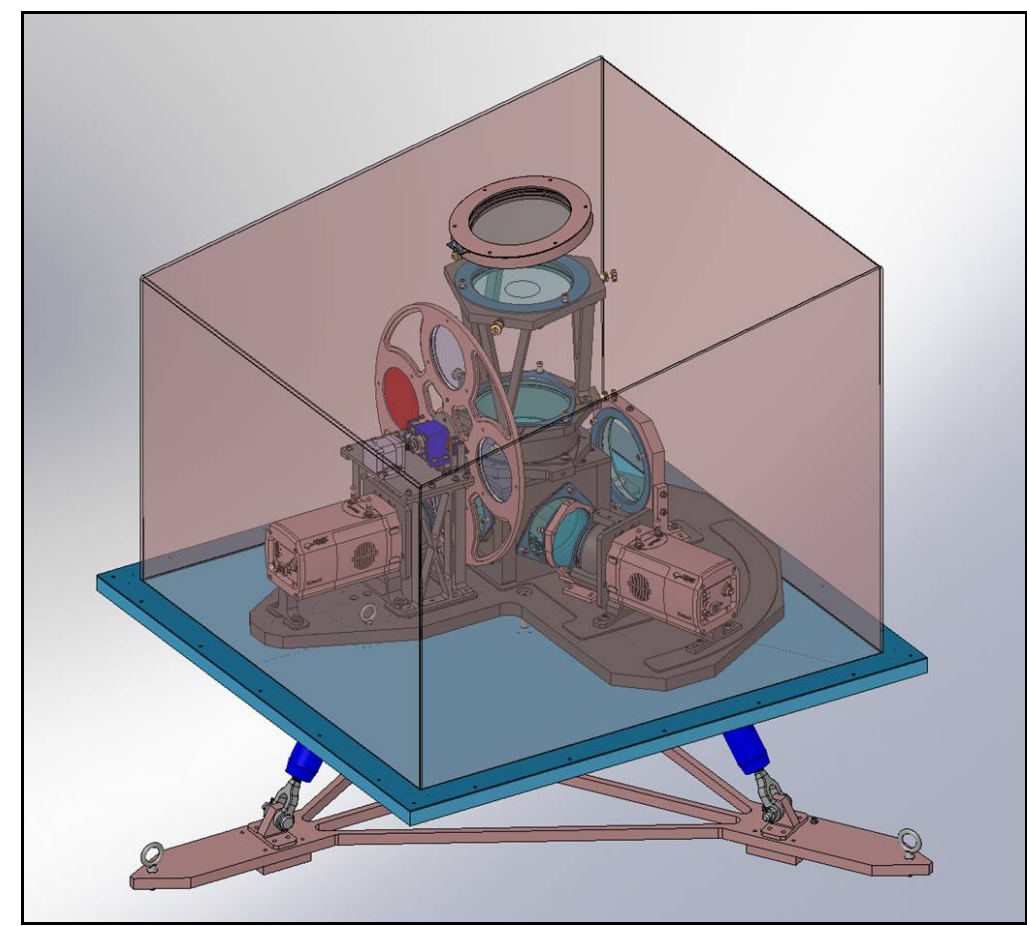

Figure 6: A mechanical rendering of the optical bench mounted to the tripod support structure with enclosure in place.

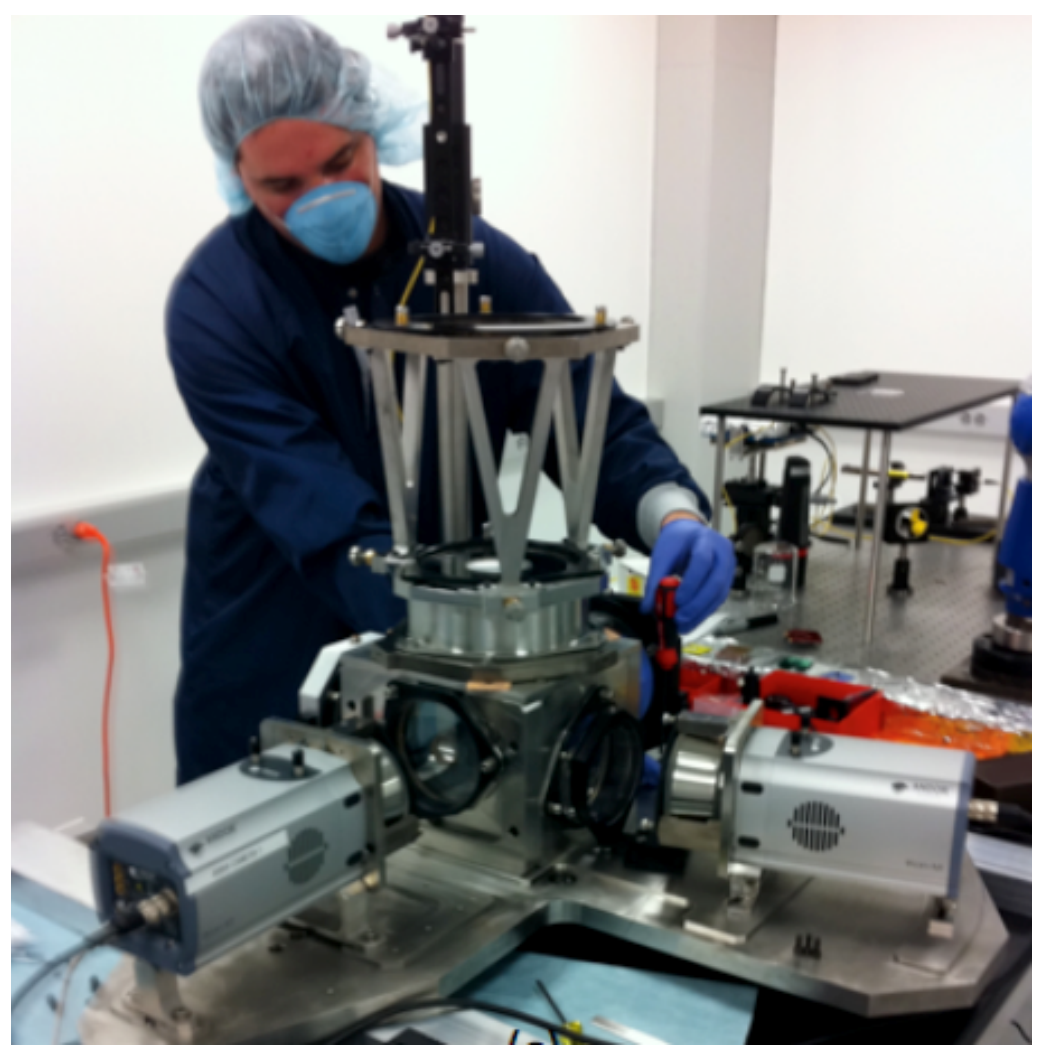

Figure 7: Assembly of the instrument at Caltech prior to deployment. 


\section{DEPLOYMENT}

\subsection{Shipment}

The instrument and all associated items such as alignment equipment were packed and driven to Port Hueneme in late December 2010 for shipment by COMAIR to the South Pole station. The crates arrived in early January 2011 and were opened and checked for damage by two team members upon arrival to the station mid January 2011. The equipment had sustained no damage during transport.

\subsection{Installation at the South Pole}

The instrument required alignment and final baffling at the South Pole. The deployment team of two requested two weeks to perform this task. The team was allocated space inside the MAPO building laboratory upon which the experiment would be eventually located to perform this task. The MAPO building is shown in Figure 8 (top left). Alignment tests were performed using a laser fed 8 " diameter Newtonian telescope as the provider of a collimated beam large enough to fill the full aperture of the Gattini SPUV system. A shear plate was used to test the collimation of the input beam. A Mercury arc lamp was used to wavelength calibrate the spectrograph channel.

Once aligned and correctly baffled the instrument was hoisted to the roof using a crane and custom designed lifting structure and positioned in the appropriate location as shown in Figure 8 (bottom left).

The instrument is operated remotely and operates autonomously with little aid required from local scientific staff. Data is transferred via the Spitter satellite system from the South Pole to Denver and is available for download to the Caltech server within 24 hours.

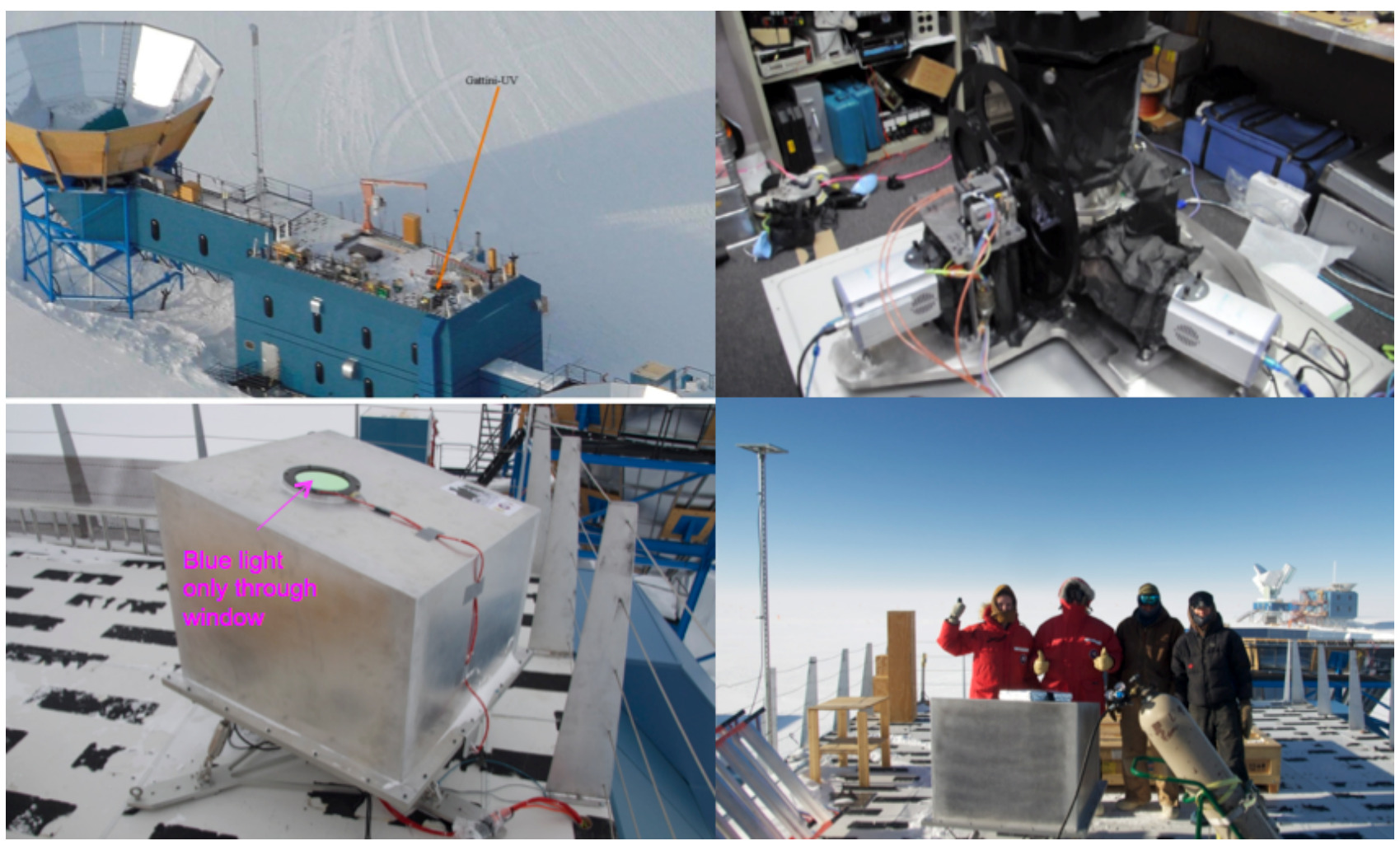

Figure 8: (top left) The Martin A Pomerantz building upon which the experiment is located, (top right) the Gattini SPUV experiment under test prior to installation on the roof, (bottom right) team members standing next to the experiment after the crane joist to the roof and (bottom left) the final location of the experiment on the MAPO roof. 


\section{PERFORMANCE}

The instrument has operated since the departure of the team in February 2011 however, it was not until April 2011 that the sky became dark enough to take meaningful data. As shown in Figure 9, the 2011 winter season defined by a solar elevation angle of less than 13 degrees begins mid April 2011 and ends in mid August 2011. There are just over four lunar cycles that occur during this period. We are most interested in quantifying the sky background during dark time hence moonless conditions. This results in approximately 8 weeks of dark time, though the instrument is operating continuously regardless of lunar or solar elevation.

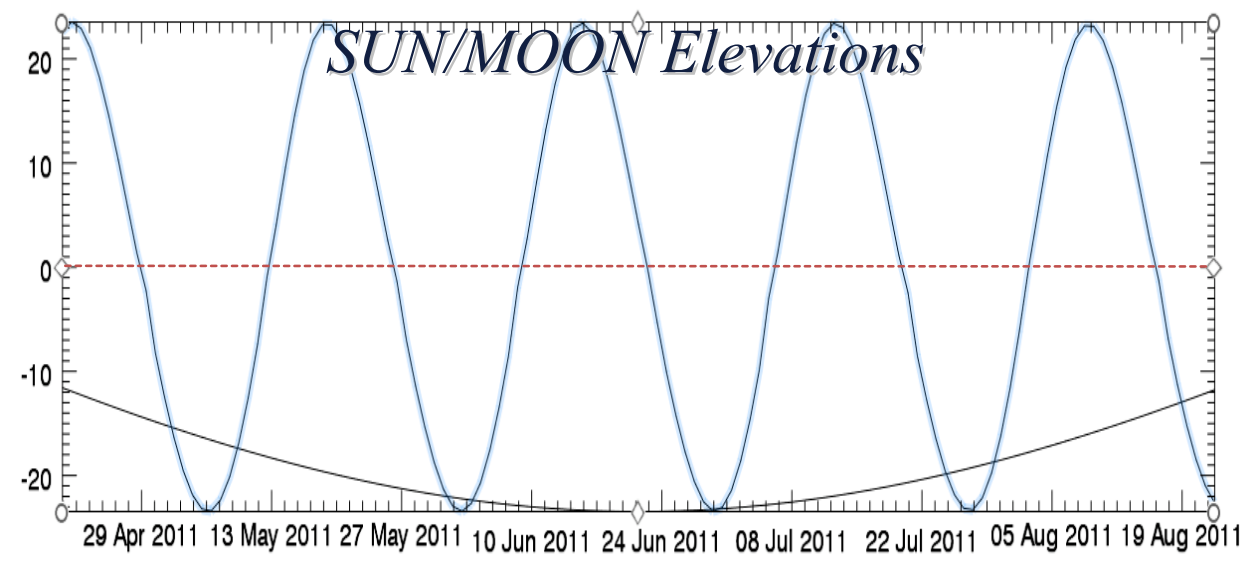

Figure 9: Solar and lunar elevation during the 2011 austral winter season. Lunar elevation is cyclic.

The instrument has operated successfully and continuously from February 2011 to present (August 2012). Annotated raw images from the imager SDSS g' filter and spectrograph channel are presented in Figure 10. Further images taken with all three filters shown with associated sky spectrum are shown in Figure 12. Figure 13 shows a magnified image of the imager channel with SDSS g' filter showing the stellar density detected. The complete 2011 dataset is currently under analysis in tandem with simultaneous cloud cover measurements derived from data taken with the All-Sky Imager experiment [4] (589nm filter, 60s exposures) taken specifically for this purpose.

We briefly list here the most important issues discovered during the commissioning period.

1. The top half of the imager field of view is slightly out of focus compared to the lower half. This is most likely caused by a misalignment of the two halves of the imager fold mirror shown in Figure 2. This does not affect the primary goals of the experiment however does limit the faintness limit of the system for continuous coverage.

2. The spectrograph slit is wider than it needs to be. The decision was made to incorporate a larger slit than can be sustained optically for ease of alignment while at the South Pole. In reality the alignment technique worked very well but removal of the installed slit was not possible. The result is a lower spectral resolution $(\mathrm{R} \sim 500)$ than designed $(\mathrm{R} \sim 2000)$.

3. Noise pick-up in the wiring connecting the filter wheel controller to the filter wheel limit switches resulted in failures of three out of the four limit switches during the 2011 season. Thankfully the system incorporated a four-fold redundancy that meant little downtime was experienced outside of a week or so for a software rewrite. The wiring was reworked during the 2011/2012 season so that three out of the four limit switches are now currently operational.

4. The power supply wire disconnected from the temperature monitoring board located inside the control pc mid season 2011. This did not disturb the experiment data collection or performance, however the camera power supplies did require changing at the end of the season due to overheating. This problem was addressed during the $2011 / 2012$ season.

Items 1 and 2 can be addressed completely by a servicing deployment in a future austral summer season. 


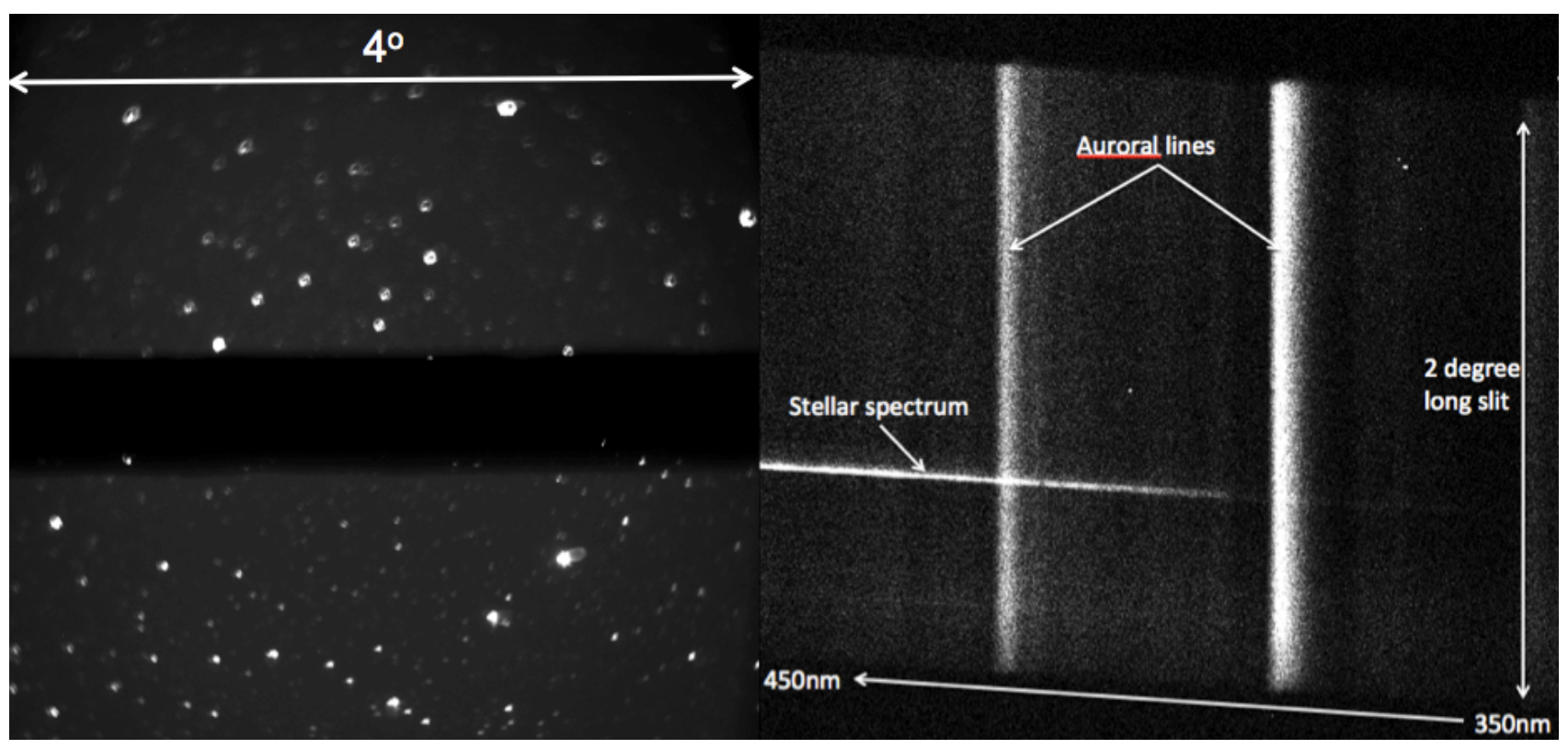

Figure 10: (left) Raw image from the Gattini SPUV imager channel with SDSS g' filter centered approximately on the South Pole. (right) Raw spectrum taken by the Gattini SPUV spectrograph channel. The dark band running centrally across the imager field of view corresponds to the field directed to the spectrograph channel.

\section{ACKNOWLEDGEMENTS}

This research is financially supported by the US National Science Foundation, the United States Antarctic Program and Caltech Optical Observtories.

\section{REFERENCES}

[1] Moore, Anna M.; Martin, Christopher; Maitless, Noam C.; Travouillon, Tony, "ACWI: an experiment to image the Cosmic Web from Antarctica", Proc. SPIE, 7012, pp. 70122A-70122A-11 (2008).

[2] Moore, Anna M.; Ahmed, Sara; Ashley, Michael C. B.; Barreto, Max K.; Cui, Xiangqun; Delacroix, Alex; Feng, Longlong; Gong, Xuefei; Lawrence, Jon; Luong-van, Daniel M.; Martin, D. Christopher; Riddle, Reed; Rowley, Nicole; Shang, Zhaohui; Storey, John W. V.; Tothill, Nick F. H.; Travouillon, Tony; Wang, Lifan; Yang, Huigen; Yang, Ji; Zhou, Xu; Zhu, Zhengxi, "Gattini 2010: cutting edge science at the bottom of the world", Proc. SPIE, 7733, pp. 77331S-77331S-12 (2010).

[3] Yuan, Xiangyan; Cui, Xiangqun; Liu, Genrong; Zhai, Fengxiang; Gong, Xuefei; Zhang, Ru; Xia, Lirong; Hu, Jingyao; Lawrence, J. S.; Yan, Jun; Storey, John W. V.; Wang, Lifan; Feng, Longlong; Ashley, Michael C. B.; Zhou, Xu; Jiang, Zhaoji; Zhu, Zhenxi, "Chinese Small Telescope ARray (CSTAR) for Antarctic Dome A", Proc. SPIE, 7012, pp. 70124G-70124G-8 (2008).

[4] Ejiri, M., M. Okada and S. Okano, All sky imager observation of aurora and airglow at South Pole Station, Antarctic Journal of the United States, XXXII, 5, 202-203, 1997. 

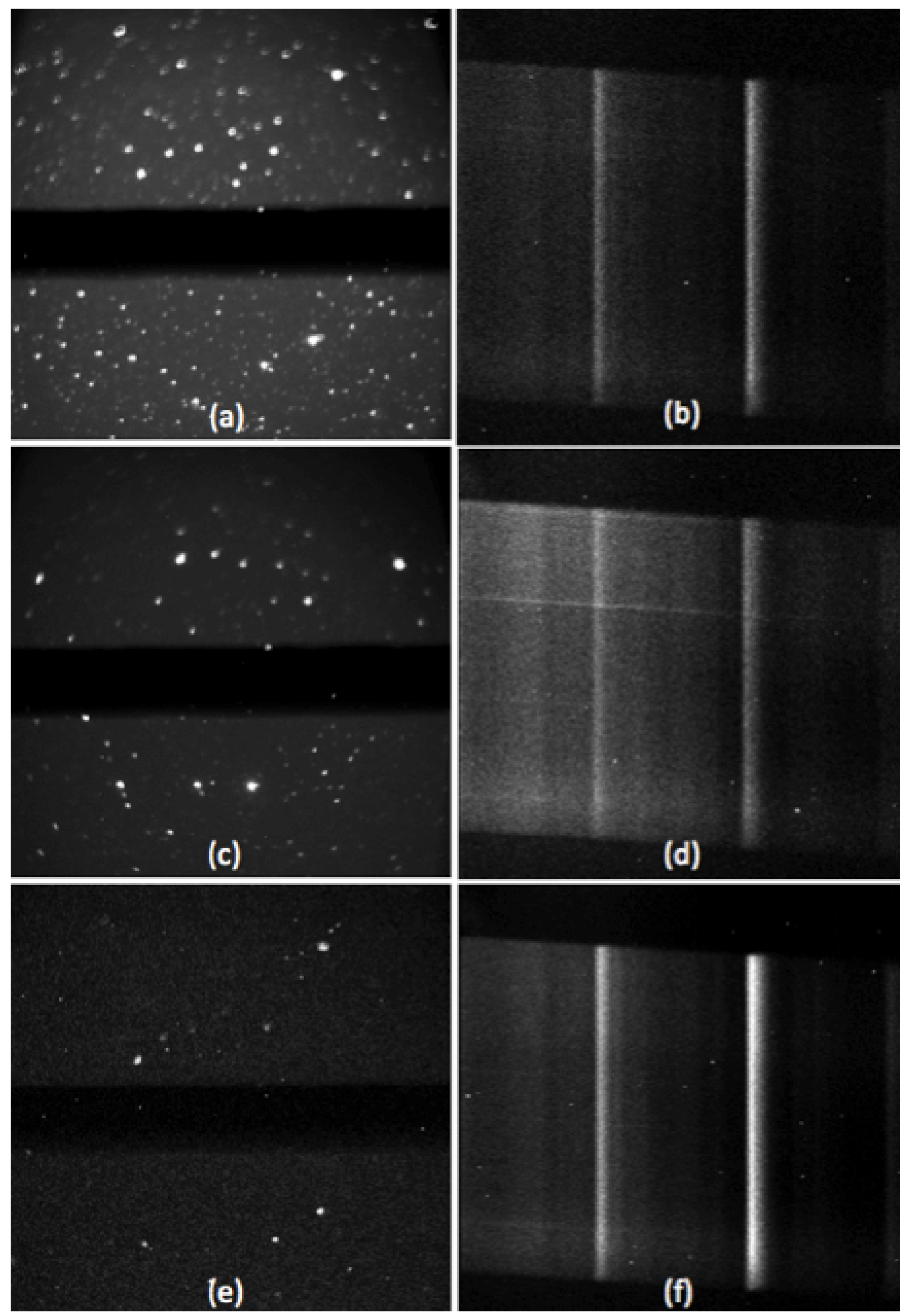

Figure 11: Raw images from the Gattini SPUV experiment taken during winter-time under moonless conditions. (a) SDSS g'filter; (b) corresponding spectrum (350nm-450nm) taken in parallel, (c) Bessel U filter image, (d) corresponding spectrum taken in parallel, (e) "Super U" filter image and (f) corresponding spectrum taken in parallel. 


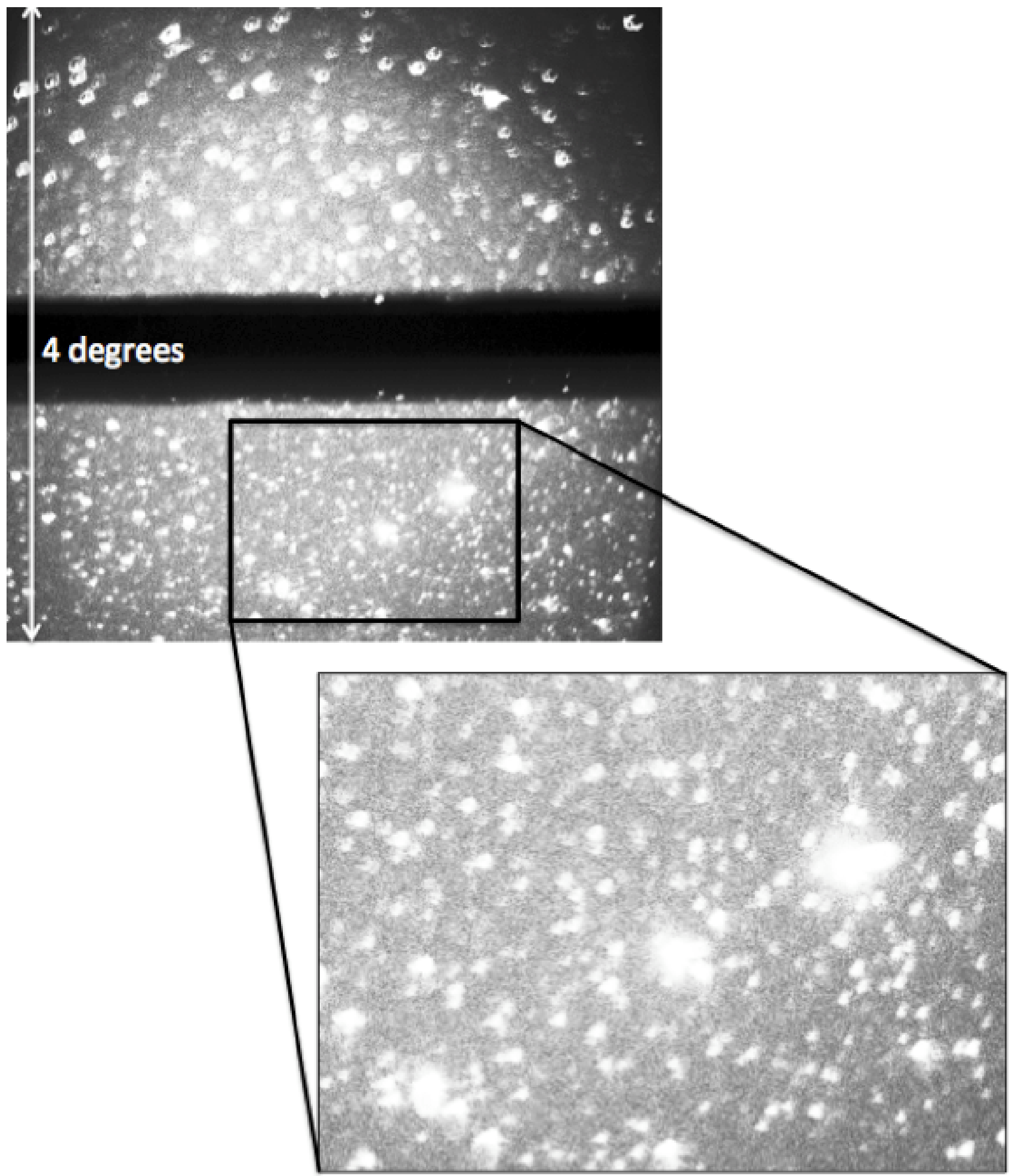

Figure 12: A magnified view of a subsection of a SDSS g' filter image taken under moonless conditions. 